\title{
Communication institutionnelle : codes de déontologie et usage spécifique de la notion de crise
}

\section{Catherine Loneux}

\section{(2) OpenEdition Journals}

Édition électronique

URL : http://journals.openedition.org/communicationorganisation/2261

DOI : 10.4000/communicationorganisation.2261

ISSN : 1775-3546

Éditeur

Presses universitaires de Bordeaux

Édition imprimée

Date de publication : 1 novembre 1999

ISSN : 1168-5549

Référence électronique

Catherine Loneux, «Communication institutionnelle : codes de déontologie et usage spécifique de la notion de crise », Communication et organisation [En ligne], 16 | 1999, mis en ligne le 27 mars 2012, consulté le 10 décembre 2020. URL : http://journals.openedition.org/communicationorganisation/ 2261 ; DOI : https://doi.org/10.4000/communicationorganisation.2261

Ce document a été généré automatiquement le 10 décembre 2020.

(C) Presses universitaires de Bordeaux 


\title{
Communication institutionnelle: codes de déontologie et usage spécifique de la notion de crise
}

\author{
Catherine Loneux
}

1 Les stratégies de communication institutionnelle visent à atteindre ce que certains auteurs ont pu appeler une "performance sociétale» (Le Moënne, 1994, p.5) du management: affirmer ou consolider la légitimité des entreprises et des différentes organisations, suggérer leur utilité sociale, rassurer sur le fait que les décisions qui y sont prises tiennent compte de l'intérêt général, contribuent au bien commun. Il est à cet égard possible de considérer que ces stratégies visent notamment à anticiper des situations critiques auxquelles pourraient se trouver confrontées diverses organisations : « organiser », n'est-ce pas d'abord, et fondamentalement, essayer d'introduire un peu de prévisibilité et d'ordre dans ce qui peut apparaître aléatoire, complexe, incertain ? La «communication de crise" (Mucchielli, 1993) se donne ainsi comme susceptible de «réduire le bruit» (Morin, 1976, p. 150) dans les situations désordonnées, mais plus largement, comme méthode de gestion standardisée et quotidienne, comme un moyen d'anticiper sur les crises en les installant littéralement au cœur de la réflexion et de l'action quotidienne des directions d'entreprises. Ne peut-on alors considérer qu'il s'agit là d'une méthode de gestion banalisée, et que le recours à la menace d'une crise est un puissant vecteur de gestion des ressources, porteur de renouveau en terme de normes notamment?

2 Deux aspects du concept de crise nous intéressent. D'abord, sa pertinence vacillante et son caractère subjectif en font un outil privilégié de la communication au sens managérial, ce que nous tenterons d'expliciter lorsque nous aborderons l'idée selon laquelle des situations de crise délibérément installées sont parfois un mode de gestion et d'autolégitimation. Ensuite, la crise étant comprise comme porteuse de perturbations et de déviances, nous verrons qu'elle stimule ainsi des processus, inventifs et créateurs, riches, qui s'entre-influent et s'entre-détruisent. En ce sens, nous proposerons l'idée d'un 
« antagonisme constructif» qui au sein des organisations est susceptible de transformer les normes en place.

3 Nous voudrions, sans prétendre qu'elle couvre l'ensemble des phénomènes qui peuvent être rassemblés sous ce vocable, finalement un peu paradoxal, de "communication de crise ", explorer plus avant cette hypothèse en examinant en particulier le rôle spécifique des discours institutionnels proposés autour de codes de déontologie professionnels : la défense de ces dispositifs d'autorégulation passe en effet souvent par une thématique de crise, identifiée par les responsables d'entreprises eux-mêmes. Notre étude nous conduira à exclure la référence transcendantale de l'éthique pour nous concentrer sur une conception des codes comme éléments émanant d'une négociation entre acteurs préoccupés par des stratégies commerciales, dans une approche pragmatique.

Nous disposons d'éléments concernant la Chambre de Commerce Internationale organisme émetteur de ces codes -, qui peuvent être perçus comme autant de traces écrites d'un discours d'auto-justification porteur d'une volonté normalisatrice. Créée en 1919 et basée à Paris, la CCI bénéficie d'une expérience éprouvée dans le domaine de la promotion des codes éthiques. Regroupant des représentants des plus grandes entreprises et présente dans des organisations internationales, elle est passée de la promotion des codes éthiques à celle d'une véritable communication institutionnelle autour de ces codes. Il s'agira d'en étudier les arguments et les thématiques qui décrètent une perte des repères, et de déceler les enjeux, en termes de régulation, contenus dans cette vision proposée de la crise. Pourquoi cette nécessité impérieuse de communiquer sur la place de l'entreprise? On peut parler de crise idéologique, qui vise à substituer les règles néo-libérales à celles de l'État. Les codes de déontologie apparaissent dans ce contexte comme vecteurs de réduction de la crise des normes imposées par les pouvoirs publics et jugées comme obsolètes. Nous nous intéresserons aux intentions stratégiques contenues dans les discours qui les portent, plus qu'à leur contenu précis. Nous tenons à préciser que par convention, lorsque nous évoquons la CCI, nous choisissons en fait d'englober dans ce vocable l'organisation entière, sa rédaction et ses acteurs d'une manière générale.

\section{La crise comme outils de gestion?}

\section{Définition flottante de la crise}

Le mot « crise » vient du grec " krisis », qui peut être traduit par « décision » (" décider » du latin «decidere » = «trancher », et « caedere» = « couper »). En ancien français « décider » = « pousser à faire », puis en latin médiéval « crisin » est employé pour décrire une maladie brutale, pour enfin atteindre la sphère politique à la fin $\mathrm{du} \mathrm{vi}^{\mathrm{e}}$ siècle. Force est de constater qu'il recouvre trois dimensions de la logique managériale: décider, organiser (mettre en ordre, en état de marche, une organisation/organisme) et gouverner (prendre le pouvoir, le garder, lancer des actions de conquête, créer des troubles en disloquant l'ordre, bref, entreprendre).

Dans un premier temps, il est utile de comprendre que la crise ne se décrète pas, qu'elle est flottante, instrumentale, et que la tentative d'installer une situation de crise participe parfois d'une visée stratégique, dans le sens où cela renverrait à une réalité se manifestant comme l'expression d'un dysfonctionnement. La crise représentera alors un point clef, un moment décisif dans tout processus de changement, désignant une 
décision, un jugement, une contestation, elle est l'événement, correspondant à l'indécision, à l'incertitude, à des blocages et des déblocages, à des antagonismes et des solidarités. Ce concept de crise pris en relation avec les changements dans les organisations nous conduira vers une définition extrêmement riche du terme, plus riche que les idées de perturbation et de désordre auxquelles il est communément associé. Les mutations cognitives peuvent être enjeux de révolution, et la régulation contient bien dans ce contexte de l'antagonisme.

7 Il y aurait crise lorsqu'apparaîtrait une modification soudaine et inattendue qui trouble le déroulement ordinaire en suscitant un état de déséquilibre. Ce n'est pas l'explosion, le surgissement du désordre qui caractérise la crise, mais la perturbation subie par l'organisation. Le désordre, la rigidification de la souplesse du système, de sa régulation et de sa stratégie, sont à considérer. La crise installe en quelque sorte le système dans une phase aléatoire. Ce cadre fournit des conditions pour une action nouvelle, pour faire basculer une organisation dans le mouvement, dans des processus désordonnés. «De même que la stratégie militaire ne peut se déployer que dans le cadre aléatoire des batailles, de même que toute situation aléatoire permet des coups d'audace dans les stratégies de jeux, y compris les jeux de la politique, de même la situation de crise, de par ses incertitudes et aléas, de par la multiplication des alternatives, crée des conditions favorables au déploiement des stratégies audacieuses et inventives, favorables à ce caractère propre à toute action: la décision entre divers comportements ou diverses stratégies possibles » (Morin, 1976, p. 160).

Toute évolution naît donc d'événements, d'accidents, de perturbations, qui donnent naissance à une déviance qui devient tendance au sein du système, explique en substance Édgar Morin. On a désorganisation/réorganisation comme évolution, l'évolution permanente étant la crise permanente. Il n'y a de théorie de la crise possible sans théorie de l'auto-réorganisation, qui se fonde sur les antagonismes au sein du système. L'antagonisme suscitant des réorganisations évolutives, le contexte de crise apparaît alors favorable à la mutation des normes. "Dès lors, tout accroissement dans une oscillation, une fluctuation, tout blocage, retard, toute insuffisance dans une régulation peuvent devenir facteurs de crise, entraînant déstructurations en chaîne... » (Morin, 1976, p. 154). Edgar Morin décrit ici une réorganisation permanente, liée à une désorganisation permanente, à la présence du désordre au sein des organisations. Aléas, désordres internes et externes se trouvent en interférence et déclenchent la crise. Le système devient incapable de résoudre les problèmes qu'il résolvait. Il est coincé, déréglé, ses normes ses règles ne fonctionnent plus.

9 En choisissant de relier la notion de crise aux altérations et ruptures que peut provoquer le changement, il s'avère intéressant d'analyser les modes de diffusion et d'acceptation des propositions alternatives, des nouveaux paradigmes. Kuhn propose, lui aussi, de penser la crise comme une condition à l'émergence de théories neuves dans le domaine de la science, avec l'approche de la notion de la «révolution scientifique » qui entraîne des changements de méthodes et de problématiques (Kuhn, 1962). Dans la période de crise, on prendrait conscience des anomalies et découvertes inassimilables par la science normale. L'état d'insécurité encouragerait l'émergence de théories rivales et disparaitrait dès l'installation d'un nouveau paradigme. La crise apparaît comme le facteur essentiel du progrès biologique, et, peut-être, en fait, de tout progrès.

Dans la communication institutionnelle, cet état d'insécurité, auquel Kuhn se réfère, apparait comme un précieux outil de gestion. Randolph Starn écrit également que le mot 
«crise » est bien « un mot clef qui reflète et illumine les intentions de ceux qui l'ont utilisé au cours des temps » (Starn, 1976, p. 18). Chez Thucydide déjà, il revêtait des significations techniques mais aussi générales, tout en étant chargé de connotations rhétoriques autant que scientifiques. Cette «malléabilité du terme» doit servir de point de départ pour admettre que parfois, « il n'y a rien d'autre que la crise elle-même dans la crise » (Stourdzé, 1976, p. 113), qu'elle est fondamentalement dans les esprits plus que dans les faits matériels. La crise est liée au sentiment que l'on est dans une situation de crise, et la communication peut servir à co-construire ce type de représentations. Elle doit pour cela savoir comprendre les approches cognitives des membres de l'organisation, être médiatrice envers eux. Il semblerait que les crises soient "des instruments qui permettent de briser les résistances », qu'elles aient un effet de démembrement sur le pouvoir. Le pouvoir prendrait lui-même la forme d'une crise, d'un mouvement critique, se définissant par les déséquilibres et les crises qu'il provoque. Le pouvoir serait «le pouvoir en miettes" (Stourdzé, 1976, p. 114). Les résistances à de nouvelles formes de pouvoir, dans cette hypothèse, se désagrégeraient à cause d'une crise, et cet état de désagrégation incarné dans la crise fixerait les conditions préalables à la déstabilisation des normes.

11 En ce sens, au-delà du processus de décrédibilisation engagé par le discours sur la crise, celui-ci affirme plus radicalement aussi la nécessité d'une mutation du rapport aux normes.

12 Anticiper les situations de crise : un mode de gestion comme mode de légitimation.

13 Cet apparent dysfonctionnement que nous venons d'évoquer, qui se manifeste par une crise des normes organisationnelles, sera pensé en lien avec la communication et l'usage qu'elle en tire. Celle-ci ne cherche-t-elle pas parfois à instaurer cette idée de crise, dans l'hypothèse où elle la mettrait elle-même sur pied comme moyen de gestion? La communication institutionnelle des organismes professionnels de type $\mathrm{CCI}$, par ses éléments discursifs et symboliques, cherche à agir sur les valeurs partagées, et encourage parfois l'émergence ou l'installation d'une crise, en n'œuvrant pas pour la déjouer mais pour l'installer au contraire afin de mieux mettre en lumière certaines valeurs. L'intérêt pour l'analyse des crises en relation avec leur effet sur les pouvoirs en place n'est ni d'observer leurs causes, ni d'examiner leurs indicateurs, mais de repérer les phases de ce processus de décrédibilisation, liées dans un premier temps aux arguments de la communication institutionnelle autour de la notion de légitimité.

Présenter la crise d'un point de vue organisationnel en offre une représentation partielle, si comme nous venons de le montrer, on voit que ce terme n'est qu'un mot sans contenu réel, un objet mal défini, sa signification ne s'associant pas forcément à celle que l'on cherche à lui attacher en la construisant. L'usage du mot crise proposé par le discours des entreprises n'est pas neutre. Si comme il l'affirme, il y a crise des pouvoirs réglementaires, sa vocation première sera de défendre la légitimité de l'entreprise par une argumentation fondée autour des croyances que chacun porte en lui, et de renforcer l'attachement aux valeurs menacées.

15 Pour assurer la légitimation d'une action qui cherche à agir sur une réglementation en place, sera considérée comme mesure légitime toute mesure qui passe pour légitime. La communication managériale produit ainsi des modèles de représentation, qui agissent sur les systèmes symboliques. L'histoire de la légitimité du secteur privé est liée à la conception pragmatique de la légitimité, qui passe par le changement et l'action et se retrouve dans le discours de communication institutionnelle. L'émergence de la notion de 
technique ou de technologie doit se concevoir dans les systèmes de légitimité traditionnels comme symptôme de la crise de légitimité, de la confusion nature/culture. Le management de la légitimité, pour l'entreprise libérale du début du siècle, a pour principe fondateur la soumission à la main invisible du marché, et la soumission à la science et à la technique - qui s'inscrivent dans la culture. Le droit est chargé de définir la légitimité de l'homo-economicus, et se situe à l'origine du pouvoir.

La légitimité est donc d'abord non-pragmatique pour l'entreprise, atomistique, qui n'est pas une organisation. Dans l'entreprise néolibérale, les organisations apparaissent, la netteté de la limitation entre le caractère privé et ce qui se passe dans l'entreprise n'existe plus. Vis-à-vis de l'extérieur, la légitimité dépend encore du droit, et vis-à-vis de l'intérieur, elle est assurée par le management scientifique. L'organisation devient complexe, sa rationalité limitée, elle ne garantit plus les mêmes principes hiérarchiques et sa relation avec la société globale change. Le système de légitimité se fait dès lors par l'articulation des mécanismes du marché, le secteur privé devenant le lieu des lois de l'économie politique. La science de la culture est alors produite sur le modèle scientifique de la nature, comme par exemple dans le domaine du respect des contrats. Pour faire régner ces lois non physiques, il faut les faire reconnaitre, les instituer, créer les conditions de leur bon fonctionnement.

Les directions d'entreprise adopteront pour cela une démarche d'auto-affirmation de leur identité propre, selon une stratégie autocentrée, autojustificatrice, dans laquelle les arguments défendent certains intérêts et altèrent les valeurs reconnues. Ce discours précis compte pour comprendre la façon dont un système de légitimité s'installe, explique Chester Barnard, qui énonce que l'autorité réside dans la personne qui reçoit l'ordre et non dans la personne d'autorité. Par là, il nous fait voir que si le discours de légitimité est accepté, c'est qu'il est en quelque sorte déjà inscrit dans l'esprit de celui qui le reçoit. Une manière de comprendre le lien qui existe entre légitimité et idéologie (Barnard, 1972).

En matière de communication autour des codes de déontologie et plus généralement autour de l'entreprise responsable, par exemple, le recours à l'argument éthique en période de crise annoncée par les pouvoirs en place permet aux responsables et représentants de l'entreprise d'opérer une autocritique, de se reconstruire, d'offrir une relecture de leur identité : "se réclamer des droits de l'homme, c'est pour un peuple, commencer par faire le récit de toutes les atteintes qu'il a portées aux droits de l'homme » (Ferry, 1998, p. 57). On peut s'ajuster ainsi aux exigences d'un espace public moderne, et la légitimité de la parole normative se fonde alors sur la reconnaissance préalable des actes qui ont contredit cette parole. Argumenter dans ce contexte revient d'un côté à contester, de l'autre, à se défendre en se justifiant rationnellement. On finalise le procès de discussion par la catégorie de l'entente à réaliser sur un énoncé valide. « $\mathrm{A}$ travers des énoncés critiquables en référence au vrai ou au faux, on met en question l'exactitude d'un fait scientifique, ou encore la justesse d'une norme juridique, et l'on discute afin d'établir ce qui est acceptable du point de vue rationnel» (Ferry, 1998, p. 159). On se met d'accord sur une forme de validité, on a un proposant, un opposant, on a une ouverture intersubjective, mais les protagonistes finissent par regarder dans la même direction.

19 L'entreprise forme un système, un ensemble d'éléments liés entre eux par des relations logiques et des résistances. Elle s'intègre dans un tout plus vaste avec lequel elle est en interaction, avec des ressources organisées, et une défaillance d'organisation peut 
entraîner une crise au sein de celle-ci. Lorsque des actions de contrôle interviennent pour éviter les perturbations, ou au contraire, pour les encourager, elles jouent sur les solidarisations de base entre les individus. Ces éléments de solidarité que nous évoquons peuvent être des règles, des coutumes, des règlements, et seront mobilisés par la communication. Elle y trouve un intérêt stratégique lui permettant à la fois de jouer sur les représentations de l'identité ou du rôle de l'entreprise présentes dans les esprits, et aussi d'anticiper voire de neutraliser certaines évolutions qu'elle juge inopportunes. Identifier la crise avant les autres, la nommer, suffirait à conférer un pouvoir, en l'occurrence, celui de fixer les modes de régulation qui régissent l'univers des professionnels de la publicité.

20 Ce discours identitaire de valorisation de l'entreprise, des organisations marchandes et de leurs normes, cherche à installer un contexte instable en donnant du sens aux choses, en les rationalisant, en construisant des référentiels par des énoncés fédérateurs, et en objectivant, enfin, pour convaincre de la nécessité d'un mode d'organisation orienté vers le changement.

\section{De la crise comme rupture : l'antagonisme constructif}

Dans un troisième temps, demandons-nous de quelle manière l'usage d'une rhétorique de crise peut-il être source de changement pour les organisations en terme de régulation, de normes, d'attribution de pouvoir. La force du discours de crise de la communication institutionnelle, qui cherche à agir sur les normes et à opérer des mutations, se situe semble-t-il dans le registre de l'événement, car «la condition d'apparition d'un événement est que se réalise un état de choses tel qu'il amènera la fin en question, ou tel que cet événement est requis pour cette fin» (Ricœur, 1985, p. 202). Paul Ricœur a montré ainsi que pour saisir les formes et l'intentionnalité de l'action, l'importance du vécu et sa mise en discours, ses diverses procédures de subjectivation, doivent être prises en compte. Parler c'est agir, c'est à dire communiquer un sens. Le concept d'événement se relie à une forme de temporalité qui privilégie l'instabilité, le chaos, et offre la possibilité d'une construction de la narration du nouveau, de la réinvention, de la propagation d'idées, de novations portées par la discontinuité qu'il engage.

Paul Ricœur propose de distinguer trois niveaux d'approche de l'événement: «1. Événement infrasignificatif ; 2. Ordre et règne du sens, à la limite non événementiel ; 3. Émergence d'événements suprasignificatifs, sursignifiants » (Ricœur, 1991, pp. 51-52). Ce troisième niveau de la sursignification retiendra notre attention, dans la mesure où il présente l'événement comme émergence, faisant partie intégrante d'une construction narrative qui engendrera elle-même un sens. La temporalisation s'avère être une composante essentielle de l'action et René Passeron évoque aussi le fait que l'événementialisation ouvre l'horizon du futur à l'imprévisibilité. Elle introduit de l'incertitude dans les projections prévisionnelles: «L'ouverture aux surprises futures introduit une béance dans la prospective » (Passeron, 1994, p. 105).

Dans cette perspective, notons que le discours identitaire proposé par la communication des entreprises, se double en effet d'un discours de déstabilisation des valeurs instituées, pour produire de l'indétermination selon un processus qui vise à rompre avec une situation connue, pour amener à une situation en devenir. Faire apparaître une crise des normes correspondra alors à un état socio-organisationnel proche de l'anomie qui n'est pas un conflit, mais une nouvelle situation donnée à partir de laquelle on recherche de 
nouveaux repères permettant à la sphère entrepreneuriale de s'arracher justement de l'anomie, ou encore à cet état d'instabilité auquel elle est prétendument confrontée.

La mutation des normes intéresse alors la communication organisationnelle dans le sens où elle dépend de processus, de dispositifs cognitifs collectifs, de référentiels, et interroge sur la manière dont les référents existent dans l'environnement des organisations, sur la façon dont ils sont perçus, dont ils évoluent dans le temps. Pourquoi affirmer ainsi qu'il y a crise donne-t-il du pouvoir à la communication qui se déploie autour de la notion de changement?

Nous assistons, semble-t-il, à des formes de normalisation généralisées, et nous pouvons nous demander comment ce que l'on nomme les « normes éthiques » réussissent à se faire accepter, comment elles imposent l'évidence d'un changement dans le rôle des acteurs de la réglementation. Cette dynamique de construction institutionnelle autour de l'éthique nous préoccupe, et nous nous situerons pour la comprendre dans le cadre des relations qu'entretiennent entre eux les professionnels, et dans celles qu'ils souhaitent proposer à la société dans un sens global et aux acteurs de la réglementation. Installer l'idée d'une crise des normes forcerait ces derniers à admettre le principe d'autorégulation, d'autocontrôle.

L'environnement institutionnel oblige les professionnels à s'adapter à un certain nombre de contraintes qui devront structurer de nouvelles interactions politiques, de nouvelles règles $\mathrm{du}$ jeu, en matière de droit notamment. Ces règles, comme ils le souhaitent, devront s'orienter de plus en plus vers des instruments d'ordre privé et se bâtiront sur des interactions entre le champ de l'action réglementaire et coercitive de l'action des pouvoirs publics et les instruments privés d'autorégulation mis en place par les agents économiques. Le processus d'harmonisation des réglementations engagé dans l'Union Européenne, par exemple, vise, au-delà d'une approche réglementaire définissant les obligations de chacun, à inciter les acteurs économiques à s'engager dans des démarches d'autocontrôle volontaires, selon des principes codifiés. Sont ainsi privilégiés les "standards ", présentés dans les codes de déontologie en matière de publicité et de marketing auxquels nous nous intéressons, mais également de manière plus globale les chartes éthiques conçues par les entreprises. L'existence de ces outils codifiés ouvre aux acteurs de nouveaux espaces de liberté.

Plus qu'au processus politique de négociation qui peut conduire à l'acceptation de ces "standards ", les modalités de mise en œuvre et d'imposition de ces normes qui passent par des discours institutionnels bien définis nous intéressent, et nous nous concentrerons sur une de leurs caractéristiques que constitue l'usage de l'idée de crise comme moyen de déstabilisation des choix réglementaires opérés traditionnellement.

Dans la démarche discursive des professionnels, l'idée de crise est utilisée pour organiser un environnement mental propre au changement. On stabilise des raisonnements, on dicte des comportements, on valorise certains thèmes. Dans une situation collective, la crise pourra mettre en évidence des contradictions, des ruptures, des désaccords, et les individus deviendront hésitants sur la ligne de conduite à tenir parce que les règles et les institutions connues apparaîtront comme déphasées par rapport aux possibilités nouvelles qu'offrent les intérêts et les idées qui surgissent du changement, sans que l'on puisse cependant se prononcer sur la justesse et l'efficacité des voies nouvelles. De l'unanimité qui existe dans un groupe autour d'un accord sur des règles existantes, on passe à l'abandon de ces règles. La déréglementation s'inscrit dans ce schéma de désorganisation, d'innovation, lié à la notion de néguentropie, qui voit que les influences 
externes peuvent encourager les crises. Le pouvoir est mis en cause, ainsi que le type de légitimité connu avec ses normes, ses valeurs, ses traditions et croyances propres.

Ce climat d'incertitude recherché, qui émerge d'une situation de crise, donnera lieu à une anticipation d'une réalité qui peut conduire à la réalisation de cette anticipation, selon l'idée de prophétie qui se réalise elle-même ("self fulfilling prophecy»), proposée par Robert Merton. «La prédiction créatrice débute par une définition fausse de la situation, qui rend vraie la conception, fausse à l'origine » (Merton, 1997, p. 139). Les événements seront artificiellement mis en avant, pour montrer que cette réalité inventée est devenue réelle, et les croyances collectives engendreront ainsi leur propre réalisation, perpétuant des jugements. Cette prédiction semble être un des processus de base de notre société, fondé sur un " conformisme anticipateur", d'acceptation des valeurs propres à renforcer une nouvelle légitimité - celle des entreprises - et à réformer les structures d'autorité. La situation sociale des hommes détermine ainsi leur conscience, leurs imaginaires et représentations collectives. La perception sociale devient le produit du cadre social, et sera porteuse des normes sociales, les individus empruntant des jugements à d'autres membres de la communauté.

Il semble en aller de même pour les entreprises et organisations. Le discours peut à cet égard être considéré comme une action. Il joue sur la légitimité en partant du postulat que de dire le droit revient à être légitime, et qu'être légitime serait une condition préalable à une transformation des normes. Affirmer d'abord qu'il y a crise, nous l'avons écrit, c'est exercer un effet sur les acteurs et délégitimer les normes en place. L'apparente incertitude des normes qui régissent les entreprises peut se comprendre en liaison avec un jeu autour de la question de la légitimité, nous l'avons expliqué plus tôt, qui passe par un discours sur la complexité et l'incertitude. Elle installe ainsi les composantes du concept de crise comme situation préalable à un changement de pouvoir. L'enjeu de la crise est bien celui du changement, puisque l'on obtient par elle de nouveaux paradigmes, une mutation théorique, cognitive, un complexe de données, d'attitudes et de valeurs, et finalement, une nouvelle source de régulation.

\section{La CCl et la logique d'événementialisation}

31 La relation spécifique entre la crise et la possibilité pour les entreprises d'agir sur les normes mérite d'être explorée: ne visent-elles pas, après avoir dénoncé les réglementations en place, à en instaurer de nouvelles? C'est là qu'apparaît notre problématique centrale. La complexification des normes, leur fragmentation, la crise de l'autorité, sont censées témoigner de la dislocation des anciens modèles de normativité. La relation entre la communication institutionnelle et la notion de crise proposée par les écrits de la CCI révèle ses intentions de renforcer son expertise, d'installer l'autodiscipline dans la durée, et s'exprime dans un registre de confrontation, de déstabilisation des mécanismes de régulation, d'événement, tentant d'introduire une rupture dans un ordonnancement donné et de faire apparaître ainsi l'urgence de changer les rapports de pouvoir.

\section{Une vocation argumentative}

32 Les acteurs de la CCI peuvent être assimilés à un "monde social ", à des agents collectifs qui ont pour objectif de produire un cadre de référence délimité par l'élaboration de 
normes éthiques, de codes de déontologie. Pour en faire la promotion, ils disposent de moyens puissants (conseil aux entreprises, groupes de travail thématiques, plate-forme pour des colloques, etc.). Essentiellement à partir d'un corpus d'articles produits par cette organisation interprofessionnelle, par des rapports d'activité, des synthèses de réunion, des actes de colloques, des résultats d'enquête, des brochures ou des notes internes destinées aux membres de ses commissions, nous examinerons comment ces multiples ressources relèvent d'une dynamique argumentative visant à promouvoir un nouvel espace social et politique. Ces instruments de légitimité professionnelle tentent d'opérer un dessaisissement des compétences et visent à rechercher une légitimité à la fois interne au sein d'un monde professionnel - l'éthique étant mise au centre de la conduite des actions -, et externe quand ils offrent une mise en scène destinée à un public plus large. Une rhétorique collective veut établir les conditions d'un accord sur de nouvelles normes.

En nous penchant sur les intentions des codes de déontologie, sur les réorganisations d'acteurs qu'ils sous-tendent, nous proposons d'envisager un contexte de crise comme pouvant renforcer l'identité commune et l'attachement à des valeurs menacées, et installer une situation favorable à la mise en place, dans un deuxième temps, de nouveaux dispositifs de pouvoir. L'argument éthique proposé par le discours institutionnel contribue souvent en effet à renforcer la stabilité des éléments de solidarité, en jouant ensuite sur ce que pourrait être la nature des règles et règlements. Où en est précisément la compatibilité entre les actions de l'entreprise et la représentation commune que le public se fait de la notion de justice réglementaire, en confrontation avec celle de sanction par l'éthique? Et quelles sont, plus concrètement, les réorganisations qui ont cours et les arguments avancés?

\section{Déontologie vs. règle de droit : vers une recomposition et un changement du rapport aux normes?}

Il nous paraît intéressant de situer les codes de déontologie dans le champ des différentes formes de régulation qui existent, afin de mieux comprendre la démarche de la communication institutionnelle qui vise à les installer. Norme, règle, régulation, procédure, norme construite, norme culturelle et sociale, norme naturelle: ces termes peuvent être définis. On observe une hiérarchie entre ces notions. Les codes de déontologie sont des normes construites, visant à l'autorégulation des organisations. Ils forment une ressource pour formaliser et justifier des actions, et pour transporter des modèles, posant la question du pouvoir comme attribut toujours à renégocier.

Le langage managérial et pragmatique émerge dans la sphère de la légitimité sociale symbolique en se mêlant aux autres formes de langage susceptibles d'établir des légitimités dans les organisations. Les emprunts à la sphère de la légitimité sociale se multiplient, pour s'assurer que le public interne et externe accepte les nouvelles représentations de la légitimité proposées. Le langage politique et des administrations s'ajoute en effet au langage managérial, évoquant la réglementation, la normalisation, etc. Certains enjeux stratégiques ne peuvent être engagés qu'avec l'accord de la sphère publique.

Pour rediscuter les normes et en induire de nouvelles, il « s'agit de réussir à installer une clôture symbolique et le contrôle par la gestion de l'assentiment " (Le Moënne, 1994, p.55). L'utilisation du terme de crise au plan discursif servira ainsi à invalider les pouvoirs réglementaires dans un premier temps, et proposera ensuite une alternative 
sous la forme de codes d'autodiscipline. Ce processus orienté vers l'installation d'un mode de régulation autocontrôlée, vers une dislocation des normes, provoque une tension entre deux formes de règles, et la communication institutionnelle développée autour des codes éthiques mobilise des référentiels nouveaux. Elle s'adresse aussi bien aux professionnels eux-mêmes qu'aux acteurs politiques et à la société d'une manière assez générale, en se nourrissant d'arguments autour d'une supposée crise des règles qui s'imposent aux entreprises, et en dénonçant les problèmes de manque de transparence dans l'exercice du pouvoir, l'irrationalité des législations en vigueur, leur mauvais fonctionnement, etc., ce afin de briser les résistances qu'opposent aux professionnels les pouvoirs en place.

Une distinction s'impose entre les «normes" et les «règles". Les normes ont un caractère d'obligation, et l'archétype de la norme est la règle de droit, qui se propage par la loi, la culture, le marché. L'interrogation sur ces catégories nous apparait pertinente lorsque l'on constate que le terme de «norme » est systématiquement présent dans les textes que nous avons analysés. Or ne devraient-ils pas avoir recours plutôt au terme de "procédure » ou de "convention"? La convention n'a pas de caractère normatif ni moral, et cependant, elle cherche à s'assimiler à une norme parfois et à agir comme telle, dans la mesure où elle s'affiche " une régularité à laquelle on croit qu'il faudrait obéir " (David, 1969, p. 97). Les codes éthiques pourraient être alors considérés comme des conventions, car ils impliquent de la part des professionnels une tentative de fixer un cadre de type conventionnaliste sur lequel ils se sont mis d'accord. La sphère des conventions passe par la médiation de la notion de normativité, passage obligé. Comment alors accorder à ces dispositifs techniques et à ces procédures normatives, élaborés dans un contexte particulier, une force normative? Nous sommes davantage en face de procédures d'interaction, et la validité des arbitrages qu'elles proposent interroge, une convention n'étant qu'un accord, un contrat passé entre deux parties.

La communication devient une dimension centrale du travail de structuration autour des normes éthiques, selon un but qui use de l'institution symbolique pour obtenir des effets de réalité. Les codes de déontologie se déclinent alors en procédures stratégiques, orientées vers la production de modèles de représentation nouveaux, qui opérationnalisent en effet la représentation commune du public vis-à-vis de la justice en proposant de nouveaux systèmes de légitimité. Aujourd'hui, notre société qui traverse une crise de légitimité, une crise des systèmes de représentation symbolique, laisse une marge ouverte à tous les discours qui profitent de cette faille. La version de la crise ici proposée ne vise qu'à déstabiliser la légitimité des règles telles qu'elles existent, pour leur substituer une alternative plus favorable aux dirigeants d'entreprises en termes de liberté d'action. Leur discours institutionnel a des effets de réalité indéniables quant à la neutralisation des pouvoirs.

Des relations se nouent entre acteurs professionnels pour un jeu d'influence visant à agir sur les règles les concernant, dénonçant leur artificialité, et les accusant de n'être que source de dysfonctionnements. À la suite du constat de ces perturbations, de nouveaux dispositifs de contrôle veulent apparaître. Les conflits peuvent exister entre individus, groupes, mais aussi entre les dispositifs de contrôle, et c'est cette forme de crise qui retient notre attention. La crise fera partie des dispositifs et stratégies de réorganisation vers des réformes, sur des bases nouvelles et de possibles restructurations. Une crise décrétée mais qui dure entraînera la recherche de solutions, tournées en l'occurrence vers une norme juridique inédite qui représentera l'innovation et réformera le système. Il 
y a donc dans toute crise un déblocage des activités intellectuelles, une contestation d'un ordre établi ou sacralisé, d'une régulation. Elle correspond à un état évolutif conçu comme transitoire auquel succède un état dit normal.

L'antagonisme est donc bien désorganisationnel, mais il constitue aussi la condition de réorganisations transformatrices et en matière de déontologie, cet antagonisme vient de l'activité de communication de la CCI qui veut agir sur la structuration de la loi. Cette situation d'urgence déclarée, espère-t-elle, entraînera avec sa fin des modifications de la figure de la régulation qui était proposée, et un changement des comportements régulateurs tournés vers l'autorégulation. Au niveau français par exemple, la CCI défend le statut des Autorités Administratives Indépendantes, inspirées dans leur fonctionnement par l'éthique professionnelle, qui constitueraient un progrès démocratique en assurant une médiation et un arbitrage entre les intérêts collectifs et le respect des droits de l'individu. Ce qui pourrait régénérer la démocratie, suspectée de fonctionner de moins en moins bien du fait de la complexité sociale et technique qui la dépasse.

\section{Éléments de discours autour de la crise des normes}

Dans cette période de crise, la CCI aurait un rôle fort à tenir. « Des dirigeants d'entreprise et des experts, membres de la CCI, établissent le point de vue des entreprises et des questions importantes de politique commerciale et d'investissement » (CCI, 09/1998, p. 8). Parmi ces sujets, on trouve entre autres celui des règles éthiques. Le fond de scène de ce discours, explique en substance Jacques Walter, est toujours celui de la crise, nous mettant en garde contre la vétusté de l'État, contre son manque d'efficacité et la perte de confiance grandissante des citoyens dans ses attributions (Walter, 1997). La CCI se propose de suppléer à ces fonctions en se posant en experte, compétente et responsable. Elle encourage les «bonnes prises de décisions", orientées contre les réglementations inefficientes des pouvoirs publics, responsables de la crise, au sens de l'entreprise.

Quelles sont plus précisément les pratiques et les représentations en jeu dans la communication offerte par les milieux entrepreneuriaux? Le primat de la transparence envers le public, celui d'une composante civique de l'entreprise, offrent une perspective universaliste de son argumentation en proposant un nouveau modèle de compétence. L'idée d'archaïsme opposée à la modernité y est omniprésente, systématiquement associée à celle d'un "renouveau nécessaire" (CCI, 09/1998, p.1). Les codes d'autodiscipline sont présentés comme une alternative salutaire face à des réglementations qui ne feraient plus face, victimes d'une perte de crédibilité, d'une désadaptation vis-à-vis de leur environnement. Ces réglementations en vigueur seraient "démodées et lentes» (CCI, 09/1998, p.1), et négligeraient le consommateur. On en appelle à une modification de l'ordre des choses, à une innovation permanente contre un ordre établi.

Le manque de dialogue entre industries et institutions générerait une situation de blocage, « difficile et improductive », si l'on en juge par les propos tenus par les membres de la CCI. L'accès à l'élaboration des textes législatifs est pour eux un droit démocratique fondamental dont le monde de l'industrie devrait bénéficier, en remplissant un rôle important pour aider à affiner et à parfaire les législations avant qu'elles n'aient un impact sur la sphère économique. Pour le cas de la France par exemple, la CCI constate que «les officiels du gouvernement tendent à regarder les problèmes dans une 
perspective politique alors que les milieux d'affaires les voient d'un point de vue économique. Les premiers sont souvent qualifiés en matière de droit public et administratif, alors que la communauté des affaires connaît mieux la discipline qui régit les affaires » (CCI, 1995 (b), p. 2). Il existe un certain scepticisme réciproque, chacun s'accusant d'incompétence, et de vision à trop court terme.

Un groupe de travail sur le renforcement des relations entre la communauté des affaires et le gouvernement a été mis sur place pour montrer l'impact positif possible des milieux d'affaires sur un processus législatif ou réglementaire. Ce document voudrait améliorer la transparence du processus législatif, réduire les mesures inutiles et contre-productives, et surtout, donner aux professionnels une image d'acteurs obligés dans tout processus de décision les concernant. La suspicion ou la crainte doivent disparaître pour laisser la place à un dialogue gouvernement/industrie, une sorte de collaboration jugée nécessaire au bon fonctionnement du système de réglementation pour éviter des législations "néfastes", «inefficaces", dont les conséquences économiques sont mauvaises et conduisent à des "impasses", des "frustrations ", des situations où seuls ceux qui ont suffisamment de pouvoir pourraient s'exprimer. Ce manque de dialogue est encore accusé de conduire à la " corruption ", au " désordre social » (CCI, 1995 (b), p. 2). Ce discours qui affirme l'existence d'une crise veut prouver une défaillance dans les mécanismes d'une régulation trop rigides, inévitable car liée à des lacunes, des imperfections, qui devraient être corrigées pour une meilleure adaptation à la réalité. On serait « face à une mosaïque de contextes légaux instables et imprévisibles ». (CCI, 1993, p.1) et l'appareil législatif mérite d'être affiné encore, cela est « crucial » (CCI, 1993, p. 3).

Les pouvoirs publics sont accusés de ne plus être "à la hauteur » face aux attentes des citoyens, entrés de plain-pied dans la "modernité » notamment en matière d'offre de médias : «l'effondrement du système entier » risque de se produire (CCI, 1995 (c), p. 3), car « les gouvernements aujourd'hui n'ont pas les ressources financières, ni le soutien des citoyens, pour créer de nouveaux médias uniquement à partir du revenu des impôts [...] les fonds apportés par l'État ne sont pas adaptés du fait de la nature de plus en plus privée et individuelle des échanges dans le cadre du développement des médias interactifs. Contrastant avec le vieux paradigme des médias de masse, les nouvelles technologies conduisent à un dialogue personnalisé avec les consommateurs. Pour que ce nouveau paradigme fonctionne - et ce le plus largement possible -, des fonds privés, dont ceux de la publicité, seront nécessaires » (CCI, 1996, p. 2). Et d'insister sur le rôle majeur de la publicité dans une économie de marché: «Les politiques au pouvoir devraient comprendre que la publicité est catalyseur pour le marché, particulièrement pour ce qui touche les nouveaux produits et services» (CCI, 1996, p. 2) La liberté de parole est présentée comme devant être épargnée par les restrictions inutiles » qui émanent parfois des pouvoirs politiques.

La CCI qualifie parfois d'elle-même de "nouveaux" certains problèmes dans ses rubriques: "Un des effets de la globalisation est que de plus en plus de problèmes autrefois pris en charge nationalement sont aujourd'hui réglés au niveau intergouvernemental. [...] (Nous faisons) face à une montée en puissance de problèmes nouveaux qui reflète la nature changeante des politiques commerciales internationales à mener » (CCI, 05/1998, p. 5) Un appel au changement qui réclame plus de simplicité dans les procédures, davantage de règles transfrontières et d'harmonisation dans les politiques à conduire, et auxquelles le milieu des affaires doit s'associer, en jouant « son rôle pour assurer qu'une approche raisonnable et équilibrée soit maintenue » (CCI, 05/1998, p. 5). 
Dans ce contexte, la CCI essaie de renforcer l'«interaction monde des affairesgouvernements ", et milite pour une attribution des responsabilités plus explicite " entre les secteurs privés et publics »; forte de son expérience en matière d'autodiscipline, elle propose des standards plus flexibles que les législations en vigueur, « mieux adaptés aux changements rapides» qui ont cours. (05/1998, p. 7). "Étendre son expertise » pour contrer des législations " prescriptives et maladroites », lutter contre « la fraude, de plus en plus complexe à comprendre car en perpétuel changement », tout dans ce discours est construit dans l'intention d'offrir une image surannée des instances traditionnelles de pouvoir trop « bureaucratiques » et « inconsistantes » (Newssheet, 1995, p. 2).

On met donc l'accent sur l'urgence de prendre en main des situations jugées préoccupantes qui requièrent une expertise technologique spécifique, mais aussi sur d'autres questions sociales nécessitant toujours des «solutions urgentes ». On trouve mention notamment d'une «crise grise» (CCI, 06/1998, p. 7), faisant allusion au vieillissement de la population dans les pays industrialisés. Elle se propose d'apporter une solution en modifiant le système des fonds de pension. Nous serions face à un "phénomène nouveau», celui de la "pression grandissante imposée par la société civile » aux entreprises pour que celles-ci assument des responsabilités qui jusqu'alors étaient du ressort de l'État. Les gouvernements, enfin, seraient devenus « impuissants à contrôler les économies nationales et à servir l'intérêt des citoyens ». L'idée selon laquelle la globalisation encouragerait le chômage, le déclin de l'industrie et l'affaiblissement de la prise en charge sociale serait fausse, et il s'agirait plutôt d'accepter l'idée d'un «changement structurel nécessaire» porté par les entreprises, seules habilitées aujourd'hui à faire face aux réalités (CCI, 06/1998, p. 8).

Globalement, l'obligation de changement est omniprésente dans les rapports, projets, notes de synthèse du travail des commissions, où les avertissements prolifèrent: les nouvelles technologies et les "promesses d'avenir» dont elles sont porteuses, les politiques d'environnement qui ne peuvent évoluer sans l'expertise scientifique des professionnels, etc. (CCI, 06/1998, p.2). En inventant une alternative aux règles actuellement en vigueur, la CCI, par sa rhétorique de crise, déstabilise réellement les repères, en installe de nouveaux, présentés comme évidents, banalisés, puisque proposés par tous « de manière courante » (CCI, 06/1998, p. 2). Sa « contribution vitale » s'impose (CCI, 04/1998, p. 1), pour encourager l'adoption d'un «éclairage nouveau» sur les schémas de régulation à adopter.

Pour conclure, nous pourrions peut-être réfléchir aux circonstances qui ont porté ce discours de crise proposant de nouvelles évaluations juridiques. Il semblerait qu'il soit lié à une situation conflictuelle, à une crise des interactions entre réseaux d'acteurs, qu'on ne peut pas expliquer de manière trop rapide, au risque d'entrer dans un processus finalisé d'évolution des rapports sociaux. On recherche à imputer des responsabilités, des responsables, on aurait des causes réductibles à l'action de groupes d'agents, de forces sociales, comme si l'action d'une force sociale donnée se déroulait dans un espace neutre, amorphe. Comme si on pouvait l'appréhender indépendamment des rapports entre cette force et d'autres forces de l'espace social. Comme si un groupe d'agents maîtrisait les conséquences sociales de ses activités. En fait, la contribution d'une force sociale au déclenchement et au devenir d'une crise ne peut être appréciée qu'à la condition que cette force ne soit pas abstraite du réseau des interactions dans lesquelles elle se manifeste et qui la façonnent. Une crise n'intervient que dans un contexte. Pour ce qui nous concerne, quel serait ce contexte ? Il pourrait se relier à une autre forme de crise qui 
inquiète les milieux managériaux, celle du rapport des individus à la norme. L'entreprise ne parvient pas à se clore de ses environnements extérieurs, et de ce fait, elle est forcée d'essayer de réduire l'écart qui existe par rapport à la norme managériale, ainsi que la dissonance entre organisations et environnements sociétaux. Il faut donc relier l'idée de crise à ses potentialités de changement pour les systèmes, les organisations et leurs processus de régulation.

\section{BIBLIOGRAPHIE}

BARNARD Chester, The Function of the Executive, Cambridge, Harvard University Press, 1972.

BEJIN André, «Crises des valeurs, crises des mesures », Communications, n² 25, Paris, Seuil, 1976, pp. 39-72.

Chambre de Commerce Internationale, Groupe de travail ad hoc sur l'emballage, le recyclage/la récupération et la gestion intégration des déchets, 10 janvier 1993, éd. CCI.

CCI, Rapport annuel, Paris, 1995 (a), éd. CCI.

CCI, Strengthening the Business-government partnership : the Need for Dialogue, Paris, 1995 (b), éd. CCI.

CCI, Business on the Internet : Interactive Marketing and Advertising, 18 juillet 1995 (c), éd. CCI.

CCI, ICC Statements on Behalf of World Business, Coommission on Marketing - Advertising and Distribution, 5 janvier 1996, éd. CCI.

CCI, Lignes directrices révisées d'ICC en matière de publicité et de marketing sur Internet, 2 avril 1998, éd. CCI.

CCI, L'organisation mondiale des affaires en 1998, mai 1998, éd. CCI.

CCI, ICC and ICC Switzerland, ICC Business Dialogue, juin 1998, éd. CCI.

CCI, Colloque spécial 75e anniversaire, Genève, 24-25 septembre 1998, septembre 1998, éd. CCI.

LEWI David K., Convention : A Philosophical Study, Cambridge, Harvard University Press, 1969.

MUNCK DE Jean et VERHOEVEN Marie (sous la direction de), Les mutations du rapport à la norme, Bruxelles, De Bœck Université, 1997, 279 p.

FERRY Jean-Marc, L'éthique reconstructive, Paris, Seuil, 1998, 115 p.

HоTIER Hugues (textes recueillis par), Éthique et communication, Bordeaux, GREC/O, ISIC, 1990, $327 \mathrm{p}$.

KUHN T.S., The Structure of Scientific Revolutions, 1962.

LAUFER Romain, L'entreprise face au risque majeur, Paris, L'Harmattan, 1972, 320 p.

MOËNNE LE Christian, Étudier les communications d'entreprises : problèmes et problématiques, HDR, Université Grenoble 3, 1994.

MERTON Robert, Éléments de théorie et de méthode sociologiques, Paris, Armand Colin, 1997, 384 p. 
MORIN Edgar, « Pour une crisologie », Communications, n² 25, Paris, Seuil, 1976, pp. 149-163.

MUCCHIELli Alex, Communication interne et management de crise, Paris, Les éditions d'organisation, 1993, 207 p.

Newssheet, juin 1995, Bruxelles, The EU Committee of the American Chamber of Commerce, 1995.

PASSERON René, Poiétique et histoire, conférence prononcée au colloque « Idée, mentalités, histoire ", Université de Sfax, Tunisie, 10 mai 1992, publiée par Espaces Temps, n55-56, 1994, p. 105.

RICCEUR Paul, Temps et récit, tome 3, Le seuil, Paris, 1985, coll. Points Seuil, 1991202 p.

STARN Randolph, « Métamorphoses d'une notion », Communications, n² 25, Paris, Seuil, 1976, pp. 4-18.

PASSERON René, « Événement et sens », Raisons pratiques, n² 2, 1991, pp. 51-52.

STOURDZE Yves, « Le pouvoir en miettes », Communications, n² 25, Paris, Seuil, 1976, pp. 113-117.

тном René, « Crise et catastrophe », Communications, n² 25, Paris, Seuil, 1976, pp. 34-38.

TIXIER Maud (sous la direction de), La communication de crise, Paris, Mc Graw Hill, 1991, 265 p.

WALTER Jacques, Le mécénat de solidarité, Communication, Frontières et mondes professionnels, HDR, décembre 1997.

\section{RÉSUMÉS}

La communication institutionnelle des entreprises autour des codes de déontologie, par ses éléments discursifs et symboliques, n'encourage-t-elle parfois l'émergence ou l'installation d'une crise en n'œuvrant pas pour la déjouer mais pour l'installer au contraire? L'hypothèse selon laquelle la défense des dispositifs d'autorégulation passe par une rhétorique de crise dont les acteurs de l'entreprise sont eux-mêmes porteurs, mérite d'être explorée. Quelle relation existe-til entre la communication institutionnelle et la notion de crise? Ce terme recouvre des significations différentes, dans le temps et selon les usages, et on le restreint ici à l'acception proposée par les discours institutionnels et selon laquelle les dispositifs de régulation seraient en crise. Sur le plan théorique, les conflits de définitions possibles et les interprétations multiples du mot crise existent, mais sont-ils pour autant à envisager ici? Ne vaudrait-il pas mieux plutôt rechercher ses origines, ses modes de développement, les possibilités et les limites de cette notion?

The corporate communication concerning professional codes of conduct, with its discursive and symbolic elements, sometimes encourage the emergence or the installation of a crisis, acting this way not to thwart it, but on the contrary to install it? The assumption that the defence of selfdiscipline Systems go through a rhetoric of crisis, that the actors of the entreprise would bear themselves, requires careful thought. What is the relation between corporate communication and the notion of crisis? This word covers different meanings, according to the periods of time and to the uses that are made of it, and I choose to restrict it to ther acceptation proposed by institutional speeches, according to which we would be in a situation of crisis. At the theorical level, conflicts of possible definitions and numerous interprétations exist, but do they have to be examined? Wouldn't it be more useful to look for the origins of this notion, its ways of development, its possibilities and limits? 


\section{AUTEUR}

\section{CATHERINE LONEUX}

Catherine Loneux est Maître de conférences au Département information-communication de l'Université Rennes 2 et membre du CERCI (Centre d'Etudes et de Recherche sur la Communication et l'Internationalisation). 